\title{
Disease-specific characteristics of vascular cell adhesion molecule-1 levels in patients with peripheral artery disease
}

\author{
Christoph Edlinger ${ }^{1}$ - Michael Lichtenauer ${ }^{1} \cdot$ Bernhard Wernly $^{1} \cdot$ Rudin Pistulli $^{2} \cdot$ Vera Paar $^{1} \cdot$ Christine Prodinger $^{3}$. \\ Florian Krizanic $^{4}$. Marcus Thieme ${ }^{3,7}$. Jürgen Kammler ${ }^{5}$. Christian Jung ${ }^{6}$ Uta C. Hoppe ${ }^{1}$ - P. Christian Schulze ${ }^{2}$. \\ Daniel Kretzschmar ${ }^{2,8}$
}

Received: 22 August 2018 / Accepted: 30 November 2018 / Published online: 7 December 2018

(c) The Author(s) 2018

\begin{abstract}
Peripheral arterial disease (PAD) is one of the most common manifestations of systemic atherosclerosis. The prevalence of unrecognized PAD is high, leading to a lack of opportunity to detect subjects at a high risk for cardiovascular events. Inflammatory processes play an important role in the disease initiation as well as in the disease progression. Vascular cell adhesion molecule 1 (VCAM-1), a biomarker of endothelial dysfunction, appears to be an important mediator in inflammatory processes. Therefore, we hypothesized that in patients with PAD, circulating VCAM-1 might be elevated due to its function in mediating adhesion of immune cells to the vascular endothelium in the process of endothelial dysfunction and inflammation, and, therefore, applicable as a diagnostic biomarker. A total of 126 non-consecutive patients were enrolled in this study, of whom 51 patients had typical clinical manifestations of PAD and as controls 75 patients with no history of PAD or cardiovascular disease. All serum samples were obtained either during hospitalization or during out-patient visits and analyzed for VCAM-1 by the ELISA. Compared with controls, median levels of VCAM-1 were significantly elevated in patients suffering from PAD (953 vs. $1352 \mathrm{pg} / \mathrm{ml} ; p<0.001)$. Furthermore, VCAM-1 appeared to be highly discriminative for the detection of PAD (AUC $=0.76$; CI 0.67-0.83). We could not observe dynamics related to increasing disease stages according to Rutherford classes in patients with apparent PAD. VCAM-1 was shown to be a potential discriminator and biomarker for the severity of systemic atherosclerosis. In a logistic regression analysis, VCAM-1 was robustly associated with the diagnosis of PAD, even after correction for clinically relevant cofounders (namely age, arterial hypertension, diabetes and LDL levels). Thusly, VCAM-1 might serve as a biomarker for PAD screening and detection.
\end{abstract}

Keywords Biomarker $\cdot$ Peripheral artery disease $\cdot$ ELISA $\cdot$ VCAM-1 $\cdot$ Cardiovascular disease

Christoph Edlinger and Michael Lichtenauer contributed equally.

Daniel Kretzschmar

Daniel.Kretzschmar@med.uni-jena.de

1 Division of Cardiology, Department of Internal Medicine II, Paracelsus Medical University of Salzburg, Salzburg, Austria

2 Department of Internal Medicine I, Friedrich Schiller University Jena, Jena, Germany

3 Department of Dermatology, Paracelsus Medical University of Salzburg, Salzburg, Austria

4 Department of Cardiology, Caritas Clinic Pankow, Berlin, Germany
5 1st Medical Department-Cardiology, General Hospital Linz, Johannes Kepler University School of Medicine, Linz, Austria

6 Division of Cardiology, Pulmonology and Vascular Medicine, Medical Faculty, University Duesseldorf, Moorenstraße 5, 40225 Düsseldorf, Germany

7 Department of Angiology/Cardiology/Diabetology, Medinos Kliniken Sonneberg, Sonneberg, Germany

8 University Clinic of Internal Medicine I, Cardiology/Angiology, Friedrich Schiller University Jena, Am Klinikum 1, 07747 Jena, Germany 


\section{Introduction}

One of the most common manifestations of atherosclerosis is peripheral artery disease (PAD), with a prevalence of around $10-25 \%$ in population aged over 55 years and an estimated total number of about 27 millions of affected patients within the industrialized world. PAD is considered to be an independent predictor of cardiac or cerebrovascular events and requires aggressive medical management [1-3]. A significant higher mortality of individuals suffering from PAD has been shown in the past $[4,5]$.

Since its first description by Brodie in 1846, a huge variety of pathophysiologic mechanisms on the development and progression of PAD have been described [6]. Over the last decades, many major clinical studies on pathogenesis of PAD focus on the role of inflammatory processes [7]. In patients with elevated inflammatory markers [e.g., CRP, lipoprotein (a), Interleukin-6 (IL-6) and soluble intercellular adhesion molecule-1 (SICAM1)], an increased risk for the development of PAD could impressively be shown in the Edinburgh Artery Study [8]. Nevertheless, the role of inflammatory parameters is still not fully unraveled, especially when it comes to potential diagnostic use or therapeutic target for medical treatments.

Today, the paramount diagnostic tool for the assessment of PAD is the ankle-brachial index (ABI) as a noninvasive tool ( $\mathrm{ABI}$ value $\leq 0.9$ in PAD). It has been shown to be a strong indicator of generalized atherosclerosis and also as a risk factor for cardiovascular diseases in general; a value $\leq 0.90$ has been shown to be associated with increased mortality [9]. The use of ABI has its limitations, including a lack of trained personnel and specialized equipment for performing proper ABI measurements in many primary practices (therefore not applied frequently in routine practice) and a high false-negative rate in patients with calcified vascular diseases, such as patients with diabetes and chronic kidney disease. This contributes to the fact that PAD still remains undiagnosed in many patients at risk, especially in earlier stages, which in turn leads to rates of "silent" PAD in up to $23 \%$ of patients $[10,11]$.

Since the diagnosis of PAD has therapeutic implications, targeting at reducing atherosclerotic vascular diseases and resulting in significant lowering of morbidity and mortality associated with PAD, early diagnosis and treatment of PAD are essential [12].

Therefore, the development of an additional/alternate diagnostic tool, particularly a biomarker-based screening test with a blood marker, is of great importance and seems appealing and feasible for the usage in PAD screening based at primary-care level. Additionally, biomarker levels may provide a more precise measurement of the extent of systemic atherosclerosis, an important determinant of the degree of functional impairment in PAD, in comparison to ABI. Further, biomarkers have already been shown to be of great help for screening, diagnostic assessment of disease severity and for follow-up and the assessment of prognosis in many other (cardiovascular) disease entities [13-19].

Over last 20 years, several inflammatory and humoral biomarkers have been identified and many studies tried to recognize a typical humoral profile or single marker as a predictor of PAD. However, many studies yielded conflicting results, and up to date, no single biomarker has been found to be a significant predictor of PAD and to be a sensitive marker for early diagnosis of the disease.

We hypothesized that vascular cell adhesion molecule 1 (VCAM-1, CD 106), a transmembrane molecule that was first described in 1989 independently by two different groups [20], and whose soluble form can be readily measured in blood, might be suitable as a candidate biomarker for PAD diagnosis.

In general, VCAM-1 is expressed within the luminal and lateral wall of endothelial cells during inflammatory processes. Within inflammation, it acts as a mediator of immune-cell adhesion to the vascular endothelium. The soluble form VCAM-1 has been shown to promote monocyte chemotaxis. Its association with numerous chronical diseases such as chronic heart failure and rheumatic could be shown in the past $[21,22]$.

Recently, an association of elevated soluble VCAM-1 levels with new onset of atrial fibrillation (AF) could be shown in a prospective, population-based cohort study with two decades of follow-up [23]. Griffin et al. could demonstrate an increasing risk for the development of AF in a population-based cohort study of older adults [24]. O'Neil et al. observed an increased risk for the development of atrial fibrillation and a consecutive increase risk for cardioembolic insults in patients suffering from PAD [25]. Together with alternate markers of inflammation, such as hsCRP and oxidative stress (e.g., MPO and NTpro-BNP), VCAM-1 has been consistently associated with PAD severity.

Up to date, no convincing investigations to clarify the role of VCAM-1 as a single predictive and diagnostic biomarker for PAD and the severity of generalized atherosclerosis, to reduce the prevalence of unrecognized PAD, have been published in literature.

We, therefore, measured VCAM-1 levels in PAD patients and tried to foster our knowledge on the association between PAD and vascular/endothelial inflammation. We hypothesized that VCAM-1 might be a potential biomarker in the screening and detection process of PAD and might consecutively contribute to identify individuals at high risk of cardiovascular events or death. 


\section{Materials and methods}

\section{Patients and controls}

In our present study, a total of 126 patients were enrolled; all were admitted to the Department of Internal Medicine I at the University hospital of Jena. 51 of these patients were diagnosed with PAD by clinical examination, apparative diagnostic (Doppler ultrasound) and functional tests (6-min walk test). 75 patients served as controls after exclusion of coronary artery disease and PAD. Patients suffering from any acute or chronic diseases, which we sought to might have a potential interaction with our hypothesis (recent history of decompensated heart failure or myocardial infarction, acute or chronic infections, malignant disease, autoimmune disease, hyperthyroidism) were excluded. Further criteria of exclusion were advanced kidney failure (glomerular filtration rate $<30 \mathrm{ml} / \mathrm{min}$.) and intake of immunosuppressive drugs. All participants gave their informed written consent before being enrolled in this study. All participants in this retrospective singlecenter study were either recruited at the Department of Cardiology or at the department of dermatology. The study protocol underwent approvement of the Ethics Committee at the Friedrich Schiller University, Jena, Germany. The study was performed in accordance to the principles of the Declaration of Helsinki (2000) and Good Clinical Practice.

\section{Blood-samples/laboratory analysis}

After a clean venous puncture in controlled haemostasis, blood samples were taken from all participants and analyzed for VCAM-1. All obtained serum samples immediately underwent storage at $-80^{\circ}$. Standard laboratory parameters (low density lipoprotein, LDL: mmol/l; triglycerides: mmol/l; high density lipoprotein, HDL: mmol/l; C-reactive protein, CRP: $\mathrm{mg} / \mathrm{l}$; creatinine: ( $\mu \mathrm{mol} / \mathrm{l})$; creatinine kinase: $(\mu \mathrm{mol} / \mathrm{l})$; BUN: $(\mathrm{mmol} / \mathrm{l})$; and blood cell count) were kindly measured and provided by the Department of Clinical Chemistry at the University Hospital Jena. Serum levels were analyzed for VCAM-1 by a commercially available enzyme-linked immunosorbent assay (ELISA) kit. (Human VCAM-1 (CD106) ELISA Development Kit, PK-EL-60062D, PromoCell GmbH, 69126 Heidelberg, Germany). All measurements were performed according to the manufacturer's instruction. In short, ninety-six-well microtiter plates were coated overnight with the capture antibody. After blocking of plates, serum samples and standard protein in different concentrations were added to the wells and incubated for $2 \mathrm{~h}$. After a washing step, a biotin-labeled anti-body was added to each well and incubated once more. Then, plates were washed again and streptavidin-horseradish peroxidase (HRP) was added. A color reaction was achieved using tetramethylbenzidine (TMB; Sigma Aldrich, USA) and it was stopped by adding a sulphuric acid stop solution (Merck, Germany). Optical density values were measured at $450 \mathrm{~nm}$ on an ELISA plate reader (Bio-Rad Laboratories, Austria).

\section{Statistical analysis}

GraphPad-Prism software (GraphPad-Software, La Jolla, CA, USA) and SPSS (22.0, SPSS Inc., USA) were used for all statistical analyses. Continuous data were expressed as mean \pm standard error of the mean (SEM) and compared with student's $T$ test. VCAM-1 was expressed as medians (with interquartile ranges) and statistically analyzed using the Mann-Whitney $U$ test. Differences between Rutherford stages were analyzed using the Kruskal-Wallis test with Dunn's post hoc test. ROC analysis was performed and area under the curve (AUC) for the determination of the diagnostic accuracy of VCAM-1 for PAD was utilized. Univariable and multivariable logistic regression analysis was used to determine whether VCAM-1 was associated with the diagnosis of PAD. For the multivariable regression model, relevant confounders (age, arterial hypertension, type 2 diabetes and LDL-cholesterol levels) were included; then, a backward variable elimination was performed. Elimination criterion was a $p$ value of more than 0.10 . A $p$ value of $<0.05$ was considered as statistically significant. Furthermore, ROC analysis was performed and an optimal cut-off was calculated by the Youden Index (as described in Ref. [26]). The overall patient cohort was retrospectively divided into two groups: those above the optimal cut-off and those below this value.

\section{Results}

Baseline characteristics are shown in Table 1. The Rutherford classification was used for disease staging. Patients within the control group were classified as Rutherford stage $0(n=55,100 \%)$. Out of all patients, diagnosed with PAD, Rutherford stage 1 was present in $0 \%$, Rutherford stage 2 in $25 \%$, Rutherford stage 3 in $47 \%$, Rutherford stage 4 in $16 \%$, and Rutherford stage 5 in $12 \%$. None of the patients had Rutherford stage 6 . The majority of patients diagnosed with PAD were males ( $82 \%$ vs. $35 \%, p<0.001)$. PAD patients were of significantly higher age than patients in the control group $(62.92 \pm 1.13$ vs. 66.78 years $\pm 1.40 ; p=0.04)$, their CRP levels were significantly elevated $(2.28 \pm 0.39$ vs. $4.79 \pm 1.23 \mathrm{mg} / \mathrm{l}, p=0.03$ ) at the time of hospital admission. Within the control group, median plasma levels of LDL (3.36 \pm 0.12 vs. $2.58 \pm 0.11 \mathrm{mmol} / 1 ; p<0.001)$, HDL 
Table 1 Baseline characteristics of the study population (PAD, nonPAD patients and overall cohort)

\begin{tabular}{|c|c|c|c|c|c|}
\hline & \multicolumn{2}{|c|}{ No PAD } & \multicolumn{2}{|l|}{ PAD } & \multirow[t]{2}{*}{$p$ value } \\
\hline & Mean & SEM & Mean & SEM & \\
\hline Age (years) & 62.92 & 1.13 & 66.78 & 1.4 & 0.04 \\
\hline BMI & 27.63 & 0.63 & 26.15 & 0.91 & 0.20 \\
\hline Ankle brachial index (ABI) & $\mathrm{n} / \mathrm{a}$ & & 0.33 & 0.03 & \\
\hline $\mathrm{CK}(\mu \mathrm{mol} / \mathrm{l})$ & 2.08 & 0.16 & 1.97 & 0.2 & 0.67 \\
\hline Cholesterin (mmol/l) & 5.55 & 0.14 & 4.8 & 0.14 & 0.00 \\
\hline $\mathrm{LDL}(\mathrm{mmol} / \mathrm{L})$ & 3.36 & 0.12 & 2.58 & 0.11 & 0.00 \\
\hline $\mathrm{HDL}(\mathrm{mmol} / \mathrm{l})$ & 1.45 & 0.05 & 1.22 & 0.04 & 0.00 \\
\hline LDL/HDL (ratio) & 2.15 & 0.17 & 2.25 & 0.14 & 0.65 \\
\hline Triglycerides (mmol/l) & 1.51 & 0.1 & 2.5 & 0.36 & 0.00 \\
\hline $\mathrm{CRP}(\mathrm{mg} / \mathrm{l})$ & 2.28 & 0.39 & 4.79 & 1.23 & 0.03 \\
\hline Thrombocytes $\left(\times 10^{9} / 1\right)$ & 229.12 & 5.83 & 214.76 & 7.31 & 0.12 \\
\hline Leucocytes $\left(\times 10^{9} / 1\right)$ & 7.08 & 0.17 & 7.42 & 0.2 & 0.20 \\
\hline Creatinine $(\mu \mathrm{mol} / \mathrm{l})$ & 74.96 & 1.94 & 79.95 & 2 & 0.08 \\
\hline BUN (mmol/l) & 5.7 & 0.22 & 5.46 & 0.3 & 0.52 \\
\hline Male & $35 \%$ & & $82 \%$ & & $<0.001$ \\
\hline $\mathrm{T} 2 \mathrm{DM}$ & $18 \%$ & & $37 \%$ & & 0.01 \\
\hline Arterial hypertension & $85 \%$ & & $90 \%$ & & 0.41 \\
\hline Fam. history for CVD & $35 \%$ & & $18 \%$ & & 0.06 \\
\hline Smoking & $30 \%$ & & $78 \%$ & & $<0.001$ \\
\hline Hyperlipidemia & $50 \%$ & & $76 \%$ & & 0.01 \\
\hline Adipositas & $45 \%$ & & $34 \%$ & & 0.023 \\
\hline \multicolumn{6}{|l|}{ Rutherford stage } \\
\hline I & $\mathrm{n} / \mathrm{a}$ & & $0 \%$ & & \\
\hline II & $\mathrm{n} / \mathrm{a}$ & & $25 \%$ & & \\
\hline III & $\mathrm{n} / \mathrm{a}$ & & $47 \%$ & & \\
\hline IV & $\mathrm{n} / \mathrm{a}$ & & $16 \%$ & & \\
\hline V & $\mathrm{n} / \mathrm{a}$ & & $12 \%$ & & \\
\hline
\end{tabular}

Patients suffering from PAD were older, had lower LDL levels and had higher CRP levels

$(1.45 \pm 0.05$ vs. $1.22 \pm 0.04 \mathrm{mmol} / \mathrm{l} ; p<0.001)$ and triglycerides $(1.51 \pm 0.10$ vs. $2.50 \pm 0.36 \mathrm{mmol} / \mathrm{l} ; p<0.001)$ were significantly higher.

Major comorbidities in the PAD group were type 2 diabetes mellitus (18\% in controls vs. $37 \%$ in PAD patients; $p=0.01$ ), hyperlipidaemia (50\% in controls vs. $76 \%$ in PAD patients; $p<0.01)$ and cigarette smoking $(30 \%$ in controls vs. $78 \%$ in PAD patients, $p<0.001$ ).

As shown in Fig. 1, VCAM-1 was significantly elevated in the PAD group (median $953 \mathrm{ng} / \mathrm{ml}$ in controls, IQR 8121218 vs. $1352 \mathrm{ng} / \mathrm{ml}$, IQR 1112-1569 in PAD patients, $p<0.0001)$. Within the PAD group, we could not observe a further rise in higher Rutherford stages. Median VCAM-1 levels according to Rutherford stage were: $1403 \mathrm{ng} / \mathrm{ml}$ in Rutherford II, $1342 \mathrm{ng} / \mathrm{ml}$ in Rutherford III, $1191 \mathrm{ng} / \mathrm{ml}$ in Rutherford IV and $1403 \mathrm{ng} / \mathrm{ml}$ in Rutherford Stage V $(p=0.74)$. VCAM-1 was highly discriminative for PAD

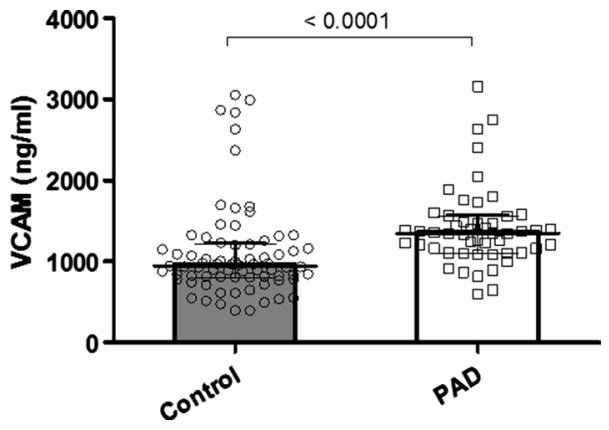

Fig. 1 Comparison of VCAM-1 levels in PAD vs. non-PAD patients

in $\mathrm{ROC}$ analysis $(\mathrm{AUC}=0.76$; $\mathrm{CI}$ 0.67-0.83). By means of the Youden Index, we calculated an optimal cut-off for the detection of PAD with a VCAM-1 level of $1079 \mathrm{ng} / \mathrm{ml}$ (Fig. 2).

Patient characteristics for patients above and below the cutoff for VCAM-1 are shown in Table 2. Interestingly, patients with a VCAM-1 level below $1079 \mathrm{ng} / \mathrm{ml}$ had higher LDL levels ( $3.38 \mathrm{mmol} / \mathrm{l}$ vs. $2.73 \mathrm{mmol} / \mathrm{l} ; p=0.00)$. We could not observe relevant differences for HDL $(1.41 \mathrm{mmol} / \mathrm{l}$ vs. $1.32 \mathrm{mmol} / \mathrm{l} ; p=0.20)$, LDL/HDL ratio (2.26 vs. 2.18 $p=0.60)$ and for triglycerides $(1.69 \mathrm{mmol} / \mathrm{l}$ vs. $2.11 \mathrm{mmol} / \mathrm{l}$; $p=0.21)$. Considering the fact that VCAM-1 is a wellknown mediator of vascular inflammation, patients with VCAM-1 levels above our cutoff value of $1079 \mathrm{ng} / \mathrm{ml}$ had unsurprisingly higher CRP levels $(3.34 \mathrm{mg} / \mathrm{l}$ vs. $1.91 \mathrm{mg} / \mathrm{l}$; $p=0.02$ ).

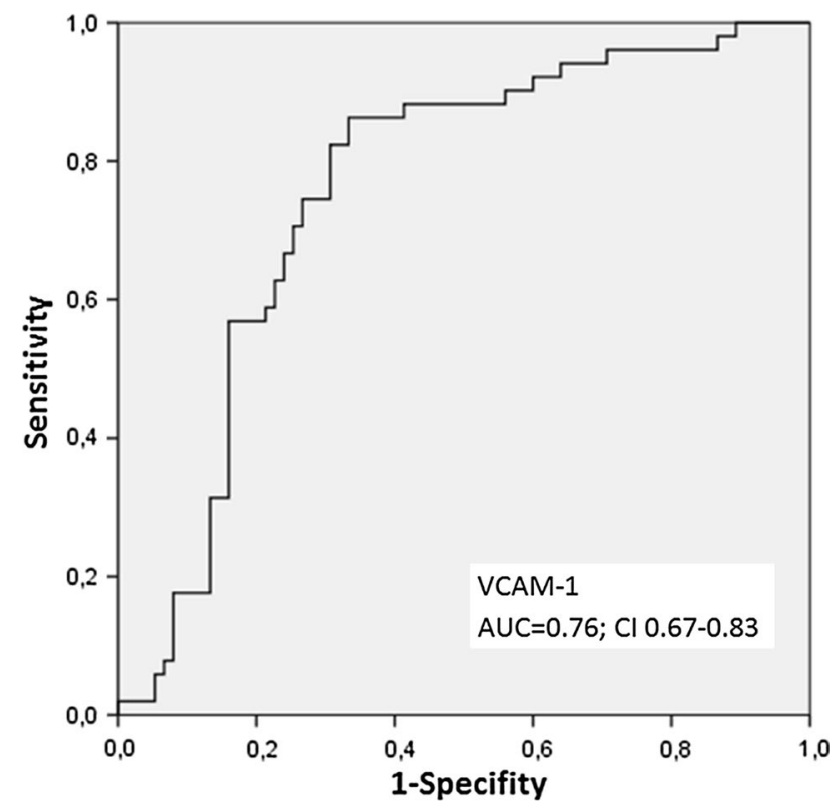

Fig. 2 ROC curve for VCAM-1 for the diagnosis of PAD. Area under the curve (AUC) for VCAM-1 was 0.76 ; CI $0.67-0.83 .44 ; p=0.01$ 
Table 2 Differences in patient characteristics between VCAM-1 levels below or above the calculated cut-off of $1079 \mathrm{ng} / \mathrm{ml}$

\begin{tabular}{|c|c|c|c|c|c|c|c|}
\hline & \multicolumn{2}{|c|}{$\begin{array}{l}\text { VCAM }<1079 \text { (ng/ } \\
\mathrm{ml})\end{array}$} & \multicolumn{2}{|c|}{$\begin{array}{l}\text { VCAM > } 1079(\mathrm{ng} / \\
\mathrm{ml})\end{array}$} & \multicolumn{2}{|c|}{ Overall cohort } & \multirow[t]{2}{*}{$p$ value } \\
\hline & Mean & SEM & Mean & SEM & Mean & SEM & \\
\hline Patients $(n=)$ & 55 & & 70 & & & & \\
\hline Age (years) & 62.69 & 10.40 & 65.98 & 8.51 & 64.39 & 9.57 & 0.07 \\
\hline BMI & 27.03 & 4.60 & 27.46 & 6.07 & 27.23 & 5.31 & 0.69 \\
\hline Ankle brachial index (ABI) & 0.32 & 0.24 & 0.33 & 0.18 & 0.33 & 0.19 & 0.91 \\
\hline Cholesterol (mmol/l) & 5.55 & 1.17 & 4.97 & 1.06 & 5.23 & 1.15 & 0.00 \\
\hline LDL (mmol/l) & 3.38 & 0.94 & 2.73 & 0.88 & 3.02 & 0.96 & 0.00 \\
\hline HDL (mmol/l) & 1.41 & 0.37 & 1.32 & 0.39 & 1.36 & 0.38 & 0.20 \\
\hline LDL/HDL (ratio) & 2.26 & 0.77 & 2.14 & 0.94 & 2.18 & 0.89 & 0.60 \\
\hline Triglycerides (mmol/l) & 1.69 & 1.02 & 2.11 & 2.28 & 1.93 & 1.84 & 0.21 \\
\hline CRP (mg/l) & 1.91 & 2.78 & 4.49 & 7.92 & 3.34 & 6.29 & 0.02 \\
\hline Thrombocytes $\left(\times 10^{9} / 1\right)$ & 234 & 50 & 213 & 50 & 222 & 51 & 0.02 \\
\hline Leucocytes $\left(\times 10^{9} / 1\right)$ & 7.39 & 1.41 & 7.05 & 1.49 & 7.20 & 1.46 & 0.21 \\
\hline Creatinine $(\mu \mathrm{mol} / \mathrm{l})$ & 76.21 & 17.91 & 77.83 & 14.15 & 77.10 & 15.91 & 0.58 \\
\hline BUN (mmol/l) & 5.65 & 1.66 & 5.55 & 2.09 & 5.59 & 1.89 & 0.78 \\
\hline
\end{tabular}

Patients with VCAM-1 concentrations above our cut-off had higher CRP levels concentrations, but significantly lower total cholesterol and HDL levels
VCAM-1 was robustly associated with diagnosis of PAD in a logistic regression model (HR $1.00195 \%$ CI $1.000-1.002 ; p=0.01)$. Even after correction for clinically relevant cofounders (age, arterial hypertension, type 2 diabetes and LDL-cholesterol levels), VCAM-1 concentration [HF 1.001, 95\% CI (1.0001-1.0018); $p=0.02$ ] remained associated with the presence of PAD.

\section{Discussion}

PAD is one of the most common manifestations of systemic arteriosclerosis, affecting about $10-25 \%$ of the general population. Due to the fact that PAD is very frequently asymptomatic, affected people often remain underdiagnosed and consecutively untreated [27]. Once PAD gets symptomatic, patients have a worse prognosis than individuals suffering from other forms of cardiovascular or cerebrovascular disease. Compared to coronary artery disease (CAD) and other cerebrovascular diseases, individuals suffering from PAD have the highest 1-year rate of atherothrombotic events [28]. The high prevalence of unrecognized PAD is largely attributable to diagnostic hurdles (e.g., infrequent use and/or false negative or increased ABI value in case of calcified vessels) that could be reduced with the introduction of specific biomarkers in screening algorithms.

The role of inflammatory processes in PAD development and PAD progression has impressively been shown in the past $[7,29]$. Many current and ongoing clinical trials in PAD focus on endothelial inflammation. Vascular cell adhesion molecule 1 (VCAM-1), a transmembrane molecule acting as a mediator of immune cell adhesion to the vascular endothelium during inflammatory processes, has been shown to be associated in chronic heart failure and rheumatic disease [21, 22]. An association with smaller calf-muscle area (resulting in a poorer lower extremity performance) and lower calf-muscle strength in PAD patients [29] and with worse performance in 6-min walk test in PAD patients with higher soluble VCAM-1 concentrations has previously been shown [30].

As we expected and in consensus with similar studies [31, 32], VCAM-1 levels were significantly increased in individuals suffering from PAD compared to controls. Interestingly, we could not observe any further increase in VCAM-1 levels in higher Rutherford classes. Our potential explanation for this finding was that individuals in higher Rutherford stages are more likely to present with clinical symptoms. Once patients are diagnosed with symptomatic PAD, their individual risk factors are usually being treated, which might have an impact on inflammatory cytokines such as VCAM-1 [31]. Eventually even lifestyle modification such as weight loss or stop of cigarette smoking might be a potential explanation for the observed stagnation in VCAM-1 levels.

As the main finding of this current study, we could show for the first time in reliable statistics that VCAM-1 levels were highly discriminative for the detection of PAD. ROC analysis revealed that at a fold change cut-off of $1079 \mathrm{ng} / \mathrm{ml}$, VCAM-1 was associated with a diagnostic accuracy of $76 \%$ in the prediction of PAD in our patients and significantly discriminate patients with PAD from those without PAD. Even after correction for clinically relevant cofounders (namely arterial hypertension, type 2 diabetes mellitus, age, LDL 
levels and cigarette smoking), VCAM-1 was still robustly associated with diagnosis of PAD in a logistic regression.

In the literature, the association of serum level of VCAM-1 and diagnosis of PAD was not consistent; several studies were limited by a small number of participants $(n<50)$ [31, 33]. Strong evidence has been established for the association of higher levels of VCAM-1 with a higher risk of PAD in patients with hemodialysis and those with diabetes [34]. A recent large study described, similar to our data, increased VCAM-1 levels in PAD patients alongside to several other inflammatory markers. However, no correlation with the clinical Rutherford stages and analysis of VCAM-1 regarding its value as a prospective biomarker has been performed [32]. We, therefore, show for the first time that VCAM-1 has potential to be used as a biomarker in the diagnosis of PAD, acting as an additive diagnostic tool to identify patients with PAD at an earlier stage of the disease thereby reducing the number of unrecognized PAD.

In the context of medical treatment, we could additionally observe an interesting finding concerning LDL-cholesterol levels. In our cohort, the optimal cut-off for the detection of PAD was $1079 \mathrm{ng} / \mathrm{ml}$. Interestingly, individuals with a VCAM-1 level below $1079 \mathrm{ng} / \mathrm{ml}$ showed significantly higher LDL-cholesterol levels. In our opinion, this finding is primarily caused by the intake of statins, which are usually prescripted, once PAD is diagnosed. This effect has also been described in an experimental analysis by Xu et al. They conclusively showed that pitavastatin reduced VCAM-1 mRNA and protein expression in TNF-alpha stimulated cultured human umbilical vein endothelial cells (HUVECs) [33]. A plethora of previous studies provided evidence for further pleiotropic effects of statins beyond their cholesterollowering effects [35]. However, anti-inflammatory mechanism and effects on endothelial homeostasis of statins have not been fully elucidated yet [36]. Moreover, also the intake of antiplatelet drugs might have influenced VCAM-1 levels. Antiplatelet therapy is recommended in all patients with symptomatic PAD [9, 37, 38]; however, in subclinical PAD, the usefulness and clinical benefit for patients remain elusive.

Based on these findings, we postulate by reinforcing previous (smaller) studies that VCAM-1 might be a promising biomarker for the detection of PAD and might contribute to a new diagnostic approach in systemic atherosclerosis, having an impact on cardiovascular mortality. However, further prospective studies are required to evaluate the clinical practicability and define the true clinical applicability of inflammatory markers in PAD detection.

\section{Limitations}

The largest limitation of this study is its single-center, sample size and retrospective layout. Due to its retrospective experimental design, we cannot provide prospective data on mortality. Further prospective studies are warranted to determine the prognostic potential of VCAM-1 as a biomarker for the diagnosis of PAD in clinical practice. Additionally, no follow-ups were performed, and therefore, the dynamic regulation of VCAM-1 in the progression of PAD could not be further assessed in this current analysis. Moreover, a possible influence of confounders such as medication on VCAM-1 levels would be of great interest; nevertheless, as no information on medication, e.g., statin intake was available, this influence cannot be ruled out. Despite these limiting factors, we believe that the current study shows the potential VCAM-1 for the diagnosis of PAD.

Acknowledgements Open access funding provided by Paracelsus Medical University.

\section{Compliance with ethical standards}

Conflict of interest The authors declare that there is no conflict of interests regarding the publication of this paper.

Open Access This article is distributed under the terms of the Creative Commons Attribution 4.0 International License (http://creativeco mmons.org/licenses/by/4.0/), which permits unrestricted use, distribution, and reproduction in any medium, provided you give appropriate credit to the original author(s) and the source, provide a link to the Creative Commons license, and indicate if changes were made.

\section{References}

1. Rooke TW, Hirsch AT, Misra S, Sidawy AN, Beckman JA, Findeiss LK, Golzarian J, Gornik HL, Halperin JL, Jaff MR, Moneta GL, Olin JW, Stanley JC, White CJ, White JV, Zierler RE (2012) 2011 ACCF/AHA focused update of the guideline for the management of patients with peripheral artery disease (updating the 2005 guideline): a report of the american college of cardiology foundation/american heart association task force on practice guidelines: Developed in collaboration with the society for cardiovascular angiography and interventions, society of interventional radiology, society for vascular medicine, and society for vascular surgery. Catheter Cardiovasc Interv 79:501-531

2. Criqui MH, Langer RD, Fronek A, Feigelson HS, Klauber MR, McCann TJ, Browner D (1992) Mortality over a period of 10 years in patients with peripheral arterial disease. N Engl J Med 326:381-386

3. O'Riordain DS, O'Donnell JA (1991) Realistic expectations for the patient with intermittent claudication. Br J Surg 78:861-863

4. Brevetti G, Martone VD, Perna S, Cacciatore F, Corrado S, Di Donato A, Di Iorio A (1998) Intermittent claudication and risk of cardiovascular events. Angiology 49:843-848

5. Leng GC, Lee AJ, Fowkes FG, Whiteman M, Dunbar J, Housley E, Ruckley CV (1996) Incidence, natural history and cardiovascular events in symptomatic and asymptomatic peripheral arterial disease in the general population. Int J Epidemiol 25:1172-1181

6. (1846) Lectures illustrative of various subjects in pathology and surgery. Med Chir Rev 4:1-22

7. Ross R (1999) Atherosclerosis - an inflammatory disease. N Engl J Med 340:115-126 
8. Tzoulaki I, Murray GD, Lee AJ, Rumley A, Lowe GD, Fowkes FG (2007) Inflammatory, haemostatic, and rheological markers for incident peripheral arterial disease: Edinburgh artery study. Eur Heart J 28:354-362

9. Aboyans V, Ricco JB, Bartelink MEL, Bjorck M, Brodmann M, Cohnert T, Collet JP, Czerny M, De Carlo M, Debus S, EspinolaKlein C, Kahan T, Kownator S, Mazzolai L, Naylor AR, Roffi M, Rother J, Sprynger M, Tendera M, Tepe G, Venermo M, Vlachopoulos C, Desormais I (2018) 2017 ESC guidelines on the diagnosis and treatment of peripheral arterial diseases, in collaboration with the european society for vascular surgery (ESVS): document covering atherosclerotic disease of extracranial carotid and vertebral, mesenteric, renal, upper and lower extremity arteriesendorsed by: The european stroke organization (ESO)the task force for the diagnosis and treatment of peripheral arterial diseases of the european society of cardiology (ESC) and of the european society for vascular surgery (ESVS). Eur Heart J 39:763-816

10. Sanna G, Alesso D, Mediati M, Cimminiello C, Borghi C, Fazzari AL, Mangrella M (2011) Prevalence of peripheral arterial disease in subjects with moderate cardiovascular risk: Italian results from the pandora study data from pandora (prevalence of peripheral arterial disease in subjects with moderate CVD risk, with no overt vascular diseases nor diabetes mellitus). BMC Cardiovasc Disord 11:59

11. Di Minno G, Spadarella G, Cafaro G, Petitto M, Lupoli R, Di Minno A, de Gaetano G, Tremoli E (2014) Systematic reviews and meta-analyses for more profitable strategies in peripheral artery disease. Ann Med 46:475-489

12. Olin JW, White CJ, Armstrong EJ, Kadian-Dodov D, Hiatt WR (2016) Peripheral artery disease: evolving role of exercise, medical therapy, and endovascular options. J Am Coll Cardiol 67:1338-1357

13. Lichtenauer M, Jirak P, Wernly B, Paar V, Rohm I, Jung C, Schernthaner C, Kraus J, Motloch LJ, Yilmaz A, Hoppe UC, Christian Schulze P, Kretzschmar D, Pistulli R (2017) A comparative analysis of novel cardiovascular biomarkers in patients with chronic heart failure. Eur J Intern Med 44:31-38

14. Lichtenauer M, Wernly B, Paar V, Rohm I, Jung C, Yilmaz A, Hoppe UC, Schulze PC, Kretzschmar D, Pistulli R (2018) Specifics of fetuin-a levels in distinct types of chronic heart failure. J Clin Lab Anal 32:e22179. https://doi.org/10.1002/jcla.22179

15. Mirna M, Wernly B, Paar V, Jung C, Jirak P, Figulla HR, Kretzschmar D, Franz M, Hoppe U, Lichtenauer M, Lauten A (2018) Multi-biomarker analysis in patients after transcatheter aortic valve implantation (tavi). Biomarkers 1-24

16. Schernthaner C, Lichtenauer M, Wernly B, Paar V, Pistulli R, Rohm I, Jung C, Figulla HR, Yilmaz A, Cadamuro J, HaschkeBecher E, Pernow J, Schulze PC, Hoppe UC, Kretzschmar D (2017) Multibiomarker analysis in patients with acute myocardial infarction. Eur J Clin Investig 47:638-648

17. Schernthaner C, Paar V, Wernly B, Pistulli R, Rohm I, Jung C, Figulla HR, Yilmaz A, Cadamuro J, Haschke-Becher E, Schulze PC, Hoppe UC, Lichtenauer M, Kretzschmar D (2017) Elevated plasma levels of interleukin-16 in patients with acute myocardial infarction. Medicine (Baltimore) 96:e8396

18. Jirak P, Mirna M, Wernly B, Paar V, Thieme M, Betge S, Franz M, Hoppe U, Lauten A, Kammler J, Schulze PC, Lichtenauer M, Kretzschmar D (2018) Analysis of novel cardiovascular biomarkers in patients with peripheral artery disease (pad). Minerva Med 15:78. https://doi.org/10.23736/s0026-4806.18.05628-8

19. Hazarika S, Annex BH (2017) Biomarkers and genetics in peripheral artery disease. Clin Chem 63:236-244

20. Osborn L, Hession C, Tizard R, Vassallo C, Luhowskyj S, ChiRosso G, Lobb R (1989) Direct expression cloning of vascular cell adhesion molecule 1 , a cytokine-induced endothelial protein that binds to lymphocytes. Cell 59:1203-1211
21. Gavrila BI, Ciofu C, Stoica V (2016) Biomarkers in rheumatoid arthritis, what is new? J Med Life 9:144-148

22. Wrigley BJ, Shantsila E, Tapp LD, Lip GY (2013) Increased expression of cell adhesion molecule receptors on monocyte subsets in ischaemic heart failure. Thromb Haemost 110:92-100

23. Willeit K, Pechlaner R, Willeit P, Skroblin P, Paulweber B, Schernthaner C, Toell T, Egger G, Weger S, Oberhollenzer M, Kedenko L, Iglseder B, Bonora E, Schett G, Mayr M, Willeit J, Kiechl S (2017) Association between vascular cell adhesion molecule 1 and atrial fibrillation. JAMA Cardiol 2:516-523

24. Griffin WF, Salahuddin T, O’Neal WT, Soliman EZ (2016) Peripheral arterial disease is associated with an increased risk of atrial fibrillation in the elderly. Europace 18:794-798

25. O'Neal WT, Efird JT, Nazarian S, Alonso A, Heckbert SR, Soliman EZ (2014) Peripheral arterial disease and risk of atrial fibrillation and stroke: the multi-ethnic study of atherosclerosis. J Am Heart Assoc 3:e001270

26. Youden WJ (1950) Index for rating diagnostic tests. Cancer 3:32-35

27. Au TB, Golledge J, Walker PJ, Haigh K, Nelson M (2013) Peripheral arterial disease-diagnosis and management in general practice. Aust Fam Physician 42:397-400

28. Steg PG, Bhatt DL, Wilson PW, D'Agostino R Sr, Ohman EM, Rother J, Liau CS, Hirsch AT, Mas JL, Ikeda Y, Pencina MJ, Goto $S$ (2007) One-year cardiovascular event rates in outpatients with atherothrombosis. JAMA 297:1197-1206

29. Beckman JA, Preis O, Ridker PM, Gerhard-Herman M (2005) Comparison of usefulness of inflammatory markers in patients with versus without peripheral arterial disease in predicting adverse cardiovascular outcomes (myocardial infarction, stroke, and death). Am J Cardiol 96:1374-1378

30. McDermott MM, Ferrucci L, Guralnik JM, Tian L, Green D, Liu K, Tan J, Liao Y, Pearce WH, Schneider JR, Ridker P, Rifai N, Hoff F, Criqui MH (2007) Elevated levels of inflammation, d-dimer, and homocysteine are associated with adverse calf muscle characteristics and reduced calf strength in peripheral arterial disease. J Am Coll Cardiol 50:897-905

31. Signorelli SS, Mazzarino MC, Di Pino L, Malaponte G, Porto C, Pennisi G, Marchese G, Costa MP, Digrandi D, Celotta G, Virgilio V (2003) High circulating levels of cytokines (il-6 and tnfalpha), adhesion molecules (VCAM-1 and ICAM-1) and selectins in patients with peripheral arterial disease at rest and after a treadmill test. Vasc Med 8:15-19

32. Signorelli SS, Anzaldi M, Libra M, Navolanic PM, Malaponte G, Mangano K, Quattrocchi C, Di Marco R, Fiore V, Neri S (2016) Plasma levels of inflammatory biomarkers in peripheral arterial disease: results of a cohort study. Angiology 67:870-874

33. Xu Q, Luan T, Fu S, Yang J, Jiang C, Xia F (2014) Effects of pitavastatin on the expression of VCAM-1 and its target gene mir-126 in cultured human umbilical vein endothelial cells. Cardiovasc Ther 32:193-197

34. McDermott MM, Liu K, Ferrucci L, Tian L, Guralnik JM, Green D, Tan J, Liao Y, Pearce WH, Schneider JR, McCue K, Ridker P, Rifai N, Criqui MH (2008) Circulating blood markers and functional impairment in peripheral arterial disease. J Am Geriatr Soc 56:1504-1510

35. Reriani MK, Dunlay SM, Gupta B, West CP, Rihal CS, Lerman LO, Lerman A (2011) Effects of statins on coronary and peripheral endothelial function in humans: a systematic review and meta-analysis of randomized controlled trials. Eur J Cardiovasc Prev Rehabil 18:704-716

36. Satoh M, Takahashi Y, Tabuchi T, Minami Y, Tamada M, Takahashi K, Itoh T, Morino Y, Nakamura M (2015) Cellular and molecular mechanisms of statins: an update on pleiotropic effects. Clin Sci (Lond) 129:93-105 
37. Gent M, Caprie steering committee (1996) A randomised, blinded, trial of clopidogrel versus aspirin in patients at risk of ischaemic events (caprie). Lancet 348:1329-1339

38. Navarese EP, Wernly B, Lichtenauer M, Petrescu AM, Kolodziejczak M, Lauten A, Frediani L, Veulemanns V, Wanha W,
Wojakowski W, Lesiak M, Ferrante G, Zeus T, Tantry U, Bliden K, Buffon A, Contegiacomo G, Jung C, Kubica J, Pestrichella V, Gurbel PA (2018) Dual vs single antiplatelet therapy in patients with lower extremity peripheral artery disease - a meta-analysis. Int J Cardiol 15:78. https://doi.org/10.1016/j.ijcard.2018.07.009 